\title{
Sufrimiento social, VIH y narrativas en torno a la muerte en mujeres trans en Lima
}

Recibido: 19/08/2019

Aprobado: 15/10/2019

\author{
ARÓN NÚNEZ-CURTO SIFUENTES \\ Universidad Nacional Mayor de San Marcos \\ aronncs@gmail.com
}

\section{RESUMEN}

Teniendo como contexto la reconstrucción etnográfica de las trayectorias de enfermedad, cuidado y fallecimiento de cuatro mujeres trans y travestis en la ciudad de Lima, este artículo busca analizar las narrativas en torno al VIH/sida, el cuidado de la salud, las maneras de afrontar la adversidad y la muerte presentes en las explicaciones y valoraciones de las mujeres trans y travestis que cuidaron de las fallecidas. Por un lado, las trayectorias ponen en discusión el impacto de la violencia institucional y las inequidades sociales en las limitadas posibilidades que tuvieron las fallecidas y sus redes de cuidado de afrontar los procesos de enfermedad producto de la infección avanzada por VIH. Asimismo, en las narrativas alrededor de lo sucedido, confluyen los efectos de una perspectiva neoliberal sobre el cuidado de la salud y una retórica del VIH/sida alrededor de la perspectiva del riesgo epidemiológico, discursos que hacen énfasis en la responsabilidad individual frente a la adversidad y la enfermedad. La narrativa sobre "la vida desordenada" es especialmente significativa, en tanto permite analizar la dinámica entre los efectos del VIH/sida como dispositivo de regulación y el estigma a la transgeneridad, como aspectos que producen sufrimiento y vulnerabilidad a mujeres trans y travestis.

Palabras clave: Mujeres trans, travestis, VIH/sida, salud, sufrimiento social.

\section{Social suffering, HIV, and narratives surrounding death among trans women in Lima}

\begin{abstract}
Based on the ethnographic study of the course of the disease, care and death of four trans women and transvestites in the city of Lima. This paper analyzes the narratives about HIV/AIDS, health care, and ways to cope with adversity and death, as part of the explanations and assessments of the trans women and transvestites who took care of the deceased. On the one hand, we analyze the impact of institutional violence and social inequities on the limited possibilities that the deceased and their care networks had to deal with the disease processes caused by HIV infection without access to treatment and care. In addition, we discuss that the effects of a neoliberal perspective on health care and a rhetoric of HIV/AIDS around the perspective of epidemiological risk are discourses articulated in the narratives analyzed. Both discourses emphasize individual responsibility as the way to face adversity and health problems. Finally, the narrative of "messy life" is especially significant, because it allow us to analyze the dynamics around the effects of HIV/AIDS as a control device, and the stigma against transgender people as a form of producing suffering and vulnerability among trans women and transvestites.
\end{abstract}

Keywords: Trans women, transvestites, HIV/AIDS, Health, social suffering. 
Mi sida me acompaña. Ríe al mismo tiempo conmigo. Se me nota. La llevo donde vaya como una capa roja. Hoy tapamos las manchas, salimos a la calle y sentimos el sol, fue un día tibio. Miramos antirretrovirales en cada vitrina [...] Mira me dice para que me detenga y ponga atención en quienes se creen inmortales. Mira como miran sin saber que también ellos mueren.

Claudia Rodríguez, poeta travesti

Nosotras, las olvidadas, ya no tenemos nombre. Es como si nunca hubiéramos estado ahí. CAmila Sosa, escritora trans, Las malas

C omenzar diciendo que una de cada tres travestis y mujeres trans ${ }^{1}$ viven con VIH en el país y que aún después de quince años de acceso gratuito al tratamiento antirretroviral siguen muriendo a causa del deterioro que produce el avance de la infección, quizá sin pasar los cuarenta años de edad (CIDH, 2015; Berkins, 2007), es una denuncia y no solo un dato epidemiológico. Y que una vez muertas, los nombres que dan testimonio de sus deseos y de quiénes fueron no aparecerán en los registros oficiales, quedando sus existencias borradas y a merced de la frágil memoria de solo aquellos/as que se resistirán a voltear la página. Esto nos convoca a pensar la investigación como resistencia y estrategia en contra de la violencia, de la indiferencia y del olvido.

Sin embargo, al mismo tiempo que lo digo, me repliego y temo por la interpretación que hagan de este hecho. Porque tal como explicaron Cosme y otros (2007), la representación circulante de la travesti, así como del homosexual, está impregnada de VIH, de contaminación, de muerte y tragedia. Mi temor es el de reforzar prejuicios fuertemente arraigados que asocian a las travestis y mujeres trans con el VIH/sida, y que reducen sus existencias a una serie de eventos lamentables que encuentran justificación en una narrativa de excesos producida activamente por los medios de comunicación, pero que impregna también otros espacios y círculos, incluso las políticas de salud. Sin embargo, considero que la investigación puede llamar la atención justamente sobre los mecanismos de exclusión a las que se enfrentan las travestis y mujeres trans, y posibilitar una escucha diferente a su sufrimiento producido activamente por las estructuras sociales (Kleinman et al., 1997).

Las ideas que presento aquí son producto de la investigación que realicé durante el año 2016 sobre trayectorias de enfermedad y muerte de mujeres

1 Uso las dos denominaciones en tanto ambas son ampliamente compartidas en el contexto peruano. Ambas dan cuenta de procesos de subjetivación y corporalización de género de personas asignadas con el sexo masculino al nacer, pero que lo largo de sus vidas construyen una identidad en el amplio espectro de lo femenino o en el tránsito continuo entre lo masculino y lo femenino. 
trans en Lima. La investigación parte de la premisa que el morir no solo constituye el desenlace de un proceso biológico de enfermedad, sino que puede ser más bien, desde una perspectiva antropológica, el punto inicial para aproximarse al carácter social del sufrimiento de una colectividad y a los mecanismos mediante los cuales se reproduce la violencia y la exclusión. La literatura antropológica sobre la salud ha demostrado que la investigación social y cultural de los procesos de enfermedad, atención y curación permiten entender los efectos que tienen las inequidades sociales, tanto en el cuerpo individual como en el social (Scheper-Hughes y Lock, 1987). Algunos estudios han explicado de qué maneras los diversos problemas que deterioran la salud de las travestis y mujeres trans se encuentran conectados con las condiciones precarias de existencia a causa de la exclusión y la violencia (Salazar et al., 2010). Así, por ejemplo, para el caso de la infección del VIH se ha demostrado que el trabajo sexual precario como estrategia de sobrevivencia, la pobreza y la discriminación en los servicios de salud son factores socioculturales, económicos y políticos que incrementan su vulnerabilidad a la infección y sus dificultades para acceder de manera oportuna y sostenida al tratamiento antirretroviral (Salazar et al., 2013, 2010). De esa manera, podemos decir que en tanto la colectividad travesti y trans tiene el más alto índice de infección el país (MINSA, 2014; Salazar et al., 2010) es una clara manifestación de lo que el antropólogo Paul Farmer (2007) llamó violencia estructural.

Si bien en este artículo haré mención de las inequidades sociales más amplias que limitan críticamente las capacidades de respuesta de las mujeres trans frente a la enfermedad, me interesa sobre todo incidir más en los procesos intersubjetivos, mediante los cuales se componen y circulan narrativas entre mujeres trans y que intentan explicar la alta carga de infección por VIH, la adversidad y la muerte. El estudio exploró, para el caso de las travestis y mujeres trans, lo que las investigaciones sobre el sufrimiento social señalan como el carácter moral del padecimiento (Ortega, 2008; Kleinman et al., 1997). Entendemos por "sufrimiento social", desde la antropología, al "ensamblaje de problemas humanos que tienen sus orígenes y consecuencias en las heridas devastadoras que las fuerzas sociales infligen en la experiencia humana" (Kleinman et al., 1997, p. 9). Conceptualizar el sufrimiento social implica no solo dar cuenta del efecto de los poderes políticos, económicos e institucionales en las vidas de la gente, sino también, simultáneamente, de cómo "estas formas de poder influyen en las respuestas [que las personas tienen frente] a los problemas sociales" (Ortega, 2008, p. 25). 
Esta línea de estudios plantea que alrededor de nuestras experiencias de padecimiento, se tejen discursos (sociales, comunitarios, individuales) que revelan valoraciones y posiciones de los sujetos frente a la adversidad. Considerando lo anterior, la salud y en particular las narrativas sobre los procesos de enfermedad y cuidado, permiten explorar en torno a los efectos de la violencia en la subjetividad.

\section{La investigación}

Este artículo presenta los principales hallazgos de un estudio realizado sobre los contextos de muerte de travestis y mujeres trans en la ciudad de Lima. ${ }^{2}$ Los casos alrededor de los cuales se realizan las interpretaciones del estudio se trataron de fallecimientos a causa de procesos de enfermedad, ${ }^{3}$ y se reconstruyeron a partir de los testimonios de las personas que cuidaron de ellas durante su padecimiento y posterior fallecimiento. En la mayoría de los casos estas personas fueron otras mujeres trans. El estudio se centró en conocer las condiciones en las cuales se desarrollaron los procesos de enfermedad, quiénes cuidaron de las enfermas y las prácticas de cuidado llevadas a cabo para afrontar estas situaciones, así como las explicaciones y relatos ofrecidos por las personas que participaron sobre estos procesos.

Durante este periodo, pude reconstruir los procesos de enfermedad y fallecimiento de cuatro mujeres trans. La perspectiva metodológica se inspira en el método de las autopsias verbales, ampliamente usado para casos de muerte materna e infantil; en especial la investigación tomó en consideración el uso del método que realiza la antropóloga Jeanine Anderson y equipo en su investigación sobre la muerte materna en ciudades de la sierra peruana (MINSA, 1999). A diferencia del uso convencional de este método que tiene como uno de sus propósitos el acopio de información para construir un diagnóstico biomédico en torno al deceso, el uso dado en este estudio tuvo como intención únicamente reconstruir el contexto e indagar en torno a las evaluaciones morales de lo sucedido. Siguiendo a Anderson y equipo (MINSA, 1999, p. 30), la reconstrucción de los casos implicó la exploración en torno a los siguientes elementos: i) hechos referidos al proceso de enfermedad, la etapa de emergen-

2 Este estudio fue presentado como mi tesis de maestría en el programa de Antropología en la Pontificia Universidad Católica del Perú, siendo mi asesora la Dra. Carmen Yon Leau. La investigación recibió el apoyo del Programa de Apoyo a la Investigación para Estudiantes de Postgrado (PAIP).

3 En este estudio solo se considera los fallecimientos por enfermedad y no por homicidio, el cual es otra causa común de muerte entre mujeres trans de acuerdo con los informes de derechos humanos. 
cia y el periodo de fallecimiento; ii) identificación de redes de cuidado y el rol jugado durante el proceso de enfermedad y posterior muerte; iii) análisis de decisiones y alternativas consideradas; iv) identificación de recursos en juego.

Los criterios para la selección de casos fueron los siguientes: i) fallecimientos que no tuvieran una antigüedad de más de tres años al 2016; ii) mujeres trans que hubieran fallecido en Lima y que el tiempo durante el cual hicieron frente a la enfermedad se hubiera desarrollado principalmente en esta ciudad; iii) redes sociales de cuidado residentes en Lima durante el tiempo que duró el trabajo de campo.

El trabajo de campo fue una experiencia con muchos retos, no solo por lo complicado y emocionalmente movilizador de conversar sobre casos de fallecimiento recientes, sino también por la dificultad de reconstruirlos. Fue desconcertante escuchar sobre tantas mujeres trans fallecidas a lo largo de los años, y sin embargo poder solo recabar información muy difusa sobre sus muertes. Se sabía en general muy poco sobre cómo habían fallecido, quiénes las habían acompañado o incluso hasta dónde fueron enterradas. Como un secreto a voces, el que muchas hayan fallecido por infecciones asociadas al VIH me era mencionado con cautela por las mujeres trans con las que conversé. Expresiones como "el bu", "el bicho" o "el que te dije" son usadas cotidianamente para no hablar directamente del VIH, a pesar de ser un problema bastante común en esta población. Sin haberlo anticipado como parte de la investigación, resultó que las cuatro mujeres trans fallecidas cuyas historias se recogen aquí murieron por infecciones asociadas al VIH. Solo una de ellas accedió al tratamiento, aunque lo descontinuó, mientras que para las otras tres fue demasiado tarde. Sus trayectorias terapéuticas, si bien no pueden generalizarse para toda la población trans en Lima, permiten analizar algunos aspectos clave para profundizar en el sufrimiento de las mujeres trans producto de vivir con (y morir por) VIH en condiciones de exclusión.

\section{Trayectorias de enfermedad y fallecimiento}

Durante la investigación, pude profundizar y reconstruir los fallecimientos de cuatro mujeres trans. Ellas fueron Claudia, falleció a los cuarenta y un años y se dedicaba al estilismo; Pamela, falleció a los treinta y tres años y era profesional de la salud; Tania y Abigail, ambas muy amigas fallecieron las dos a los treinta años, y se dedicaban al trabajo sexual. Las cuatro fallecieron de infecciones asociadas a una etapa avanzada del VIH. 
Un aspecto compartido en sus procesos de enfermedad es que todas vivieron su diagnóstico de VIH en secreto, y posiblemente tres de ellas sin acceder al tratamiento antirretroviral. Solo en el caso de Claudia se conoce que accedió al tratamiento, sin embargo, no se sabe con claridad su nivel de adherencia al régimen de medicación. Dado que las cuatro fueron muy cautelosas sobre la gravedad de su enfermedad, la información que se tiene sobre sus procesos de enfermedad y malestares son difusos. Solo en la etapa de emergencia, cuando el estado de salud desmejoró considerablemente necesitándose así la intervención médica, es que más personas intervinieron en el cuidado. Por tal motivo, la reconstrucción de sus historias se centra principalmente en esta etapa, la que además en ninguna de las cuatro trayectorias excede a un año.

Las enfermedades, para el caso de las mujeres trans con las que conversé durante el estudio, fueron vividas sin que constituyeran un acontecimiento relevante hasta que los malestares les impidieron trabajar, haciéndose visible para los demás. Enfermedades respiratorias, dolores corporales, dolores de cabeza, malestares producto de infecciones de transmisión sexual, emergen de las conversaciones sobre la salud y son asociados al trabajo sexual callejero y a la rutina de vida que impone este medio de subsistencia.

En las cuatro historias, el ocultamiento del VIH ha sido un punto en común entre ellas. Las personas asociadas al cuidado de las mujeres trans entrevistadas mencionaron que la vergüenza fue la causa para vivir la infección en secreto y sin apoyo. A partir de un análisis de las trayectorias, este ocultamiento tuvo dos consecuencias importantes a considerar en la salud de las mujeres trans fallecidas. Por un lado, impidió el acceso oportuno al tratamiento antirretroviral, es decir, fue una barrera para controlar la infección e impedir así que enfermedades oportunistas graves se desarrollen. De otro lado, el ocultamiento impidió los cuidados de sus amigas y allegados, que podrían haber servido de soporte — físico como emocional — para enfrentar mejor el vivir con el virus. Por tal motivo, en los cuatro casos, las redes de cuidado se activaron principalmente durante la emergencia, cuando la salud física se vio fuertemente agravada, viéndose reducida así las posibilidades de sobrevivir.

\section{Comunidad moral, vergüenza y exclusión}

A lo largo del trabajo de campo, las travestis y mujeres trans entrevistadas manifestaron, de distintas maneras, que por ser trans sus esperanzas de vida eran 
limitadas, y que se podían morir pronto debido a las condiciones de existencia marcadas por la inequidad y la violencia. Ya sea en las bromas que Tania y Abigail hacían, tal como recuerdan algunas de sus amigas, sobre "quién va a enterrar primero a la otra", o en comentarios sobre la posibilidad de contraer el VIH por una relación sexual sin protección, la posibilidad de morir estuvo tan presente en la vida cotidiana de las mujeres trans con las que conversé para este estudio, como lo estaba para las madres de la favela de Alto do Cruzeiro en Brasil, que relata Nancy Scheper-Hughes (1997) en su conocida etnografía sobre la mortalidad infantil.

Frente a la adversidad, una alternativa aprendida en condiciones de exclusión es mitigar y ocultar la propia vulnerabilidad presente en estados de salud frágiles, como un recurso de fortaleza y una estrategia de sobrevivencia, tal como se expresa en la siguiente cita:

No sé cuánto tú debes haber hablado con otras personas, o cuán consciente estás de los riesgos que se asumen en este modo de expresarte, de sentirte y de vivir. O sea, hay bastantes riesgos que tomas, y uno sabe qué es lo que está tomando, y aun así le vas para adelante. No le arrugas a la vida [...], te endureces, te forma una autosuficiencia en algún aspecto, pero en otro te hace débil [...]. Justo por esa debilidad puede afectarte en tu existencia (Sandra, mujer trans).

En un estudio sobre narrativas del dolor en la experiencia de encarnar la transgeneridad en travestis de Recife, la antropóloga Adriana Figueiredo (2011) llama "ethos de coraje" a un marco de sentido desde el cual se interpretan las experiencias de dolor y sufrimiento. Siguiendo a Figueiredo, podría argumentarse que en las condiciones de exclusión vividas se constituye "un ethos de coraje" frente a la idea de una muerte temprana, la cual tal cómo explica Sandra también es un factor que vulnerabiliza a las mujeres trans.

De acuerdo con los antropólogos Arthur y Joan Kleinman (1997), es posible identificar en los grupos sociales ciertos patrones en las formas de sobrellevar los problemas y dificultades, los cuales son transmitidos y aprendidos, directa o indirectamente. A esto ellos le llaman "modos colectivos de experiencia", los cuales dan forma a las percepciones y expresiones individuales en torno al dolor y a la aflicción (Kleinman et al., 1997). Así, el testimonio de Sandra nos introduce a un modo colectivo de afrontar la adversidad y a un sentido de "comunidad moral" a través de esta actitud compartida de coraje.

Es importante puntualizar que la noción de comunidad moral usada desde esta perspectiva teórica no atañe a un grupo constituido de sujetas/os en 
un territorio determinado. En este caso, el concepto trata de las relaciones "donde se llevan a cabo y encuentran el sustento aquellos juegos de lenguaje que constituyen una forma de vida, donde se define el repertorio de plausibles enunciados y acciones, donde se encuentran los recursos socioculturales con que las personas enfrentan a la adversidad" (Ortega, 2008, p. 24). En ese sentido, más que un lugar de significados trascendentes, la comunidad moral refiere a las relaciones y vínculos a través de los cuales se llevan a cabo prácticas que instituyen significados compartidos y que ponen a disposición recursos para la vida misma.

Si consideramos que la "comunidad moral" es aquella en donde las experiencias de vida individuales encuentran su sentido colectivo a través de un repertorio de significados compartidos y de expectativas sociales implícitas, será importante analizar qué pudo significar la "vergüenza" para las travestis y mujeres trans que vivieron en secreto su proceso de enfermedad. La respuesta que intentamos brindar aquí busca, sobre todo, indagar el contexto social y moral en el que fueron tomadas las decisiones que llevaron a las mujeres trans fallecidas a enfrentarse, como lo hicieron, al hecho de vivir con VIH.

Martha Nussbaum (2006, p. 28) señala que "la vergüenza está por lo general relacionada con ideales o normas serias y, por lo tanto, es siempre moral en un sentido amplio del término". Un aspecto importante que apunta Ortega (2008) en relación con la comunidad moral, y que tiene que ver con el lugar de la vergüenza en la discusión que nos ocupa, es que en ella operan regulaciones, las cuales asignan pertenencia y seguridad a sus miembros en base a "acuerdos vividos". Las regulaciones implican también no reconocer o deslegitimar a algunos de sus miembros y con ello "permite, autoriza o genera dinámicas de destrucción y sufrimiento social" (Ortega, 2008, p. 24).

Durante el trabajo de campo, me explicaron cómo es frecuente que entre las mujeres trans se rumoree sobre quién tiene VIH. De las que se piensa que puedan tener, se las llama — de manera socarrona_ "muertitas". Viviana, una trabajadora sexual trans del Cercado de Lima, explica el estigma alrededor del VIH, el que además de causar temor es también un motivo de vergüenza:

Porque tienen vergüenza de contar. Acá existe entre las chicas trans - dicen: "Ay, no, qué voy a tener esa enfermedad" - una discriminación para esa enfermedad [...]. Dicen: “Ay, qué voy a tener esa enfermedad. Ni loca. Buses no...”. $\mathrm{Y}$ las que dicen más son las que tienen, pero no lo dicen porque tienen vergüenza que se burlen de ella, es por eso (Viviana, mujer trans). 
Los rumores del "secreto a voces" que es vivir con VIH entre mujeres trans se vuelven así un canal a través del cual se pone en marcha el proceso estigmatizador. Aquí es importante tener en cuenta las implicaciones del estigma (en este caso referido al VIH) en la producción de una comunidad moral. Goffman (1995) considera que quienes estigmatizan buscan con ello ponerse a salvo de aquello que asusta y contamina. Mediante el proceso de estigmatización expresado en burlas y apelativos, que hacen mención a la muerte y a la enfermedad, se pretende construir una normalidad "sana" y "viva", una paradójica seguridad frágil ante la vulnerabilidad al VIH que se vive de manera cotidiana, pero de la cual no se habla. Es interesante considerar, como sugiere Nussbaum (2006, p. 257), que "la normalidad es [...] una buena manera de ocultarse", es decir, ocultar la propia vulnerabilidad.

Resulta importante mencionar que la valoración del VIH asociada a la muerte y la vergüenza es producto también de una respuesta nacional a la prevención de la infección que ha descuidado las condiciones estructurales, en especial para las comunidades más afectadas. De igual manera, un sentido común que asocia el VIH con la muerte y el miedo no ha sido desarticulado eficientemente, y sigue impregnando los mensajes preventivos que se difunden.

Considerando esta situación estructural, es necesario analizar las particularidades para el caso de las mujeres trans. El proceso estigmatizador asociado a vivir con VIH pone de manifiesto las relaciones de diferenciación social que se establecen entre mujeres trans, y que impactan negativamente en el cuidado de la salud (Menéndez, 2009). El factor de clase, que además de vinculado al acceso económico se vincula fuertemente con el capital cultural y las expectativas de ascenso social, es muy importante de considerar.

\section{Los esfuerzos y las limitaciones del cuidado de las mujeres trans enfermas}

La antropología ha evidenciado cómo el cuidado de la salud es un asunto eminentemente social y cultural. A través de la descripción de los itinerarios terapéuticos de las mujeres trans se pueden identificar a las personas que conforman sus redes de cuidado, los contextos en los que el cuidado tuvo lugar, los recursos a los que accedieron (incluyendo los institucionales, como la atención médica) y las prácticas de cuidado en la cotidianidad. 
La reconstrucción de los itinerarios ha mostrado el papel crucial que cumplieron otras mujeres trans allegadas a la fallecida para el cuidado de su salud. Esto contrasta con la ausencia de la familia, institución cuya relación con la violencia hacia las mujeres trans ya ha sido explorada en otros estudios (Jaime, 2011; Salazar et al., 2010, 2009).

El cuidado ofrecido se articuló en distintos tipos de prácticas, que fueron llevadas a cabo en momentos diferentes de la trayectoria de enfermedad, por ejemplo, la asistencia cotidiana en tareas domésticas, organización de actividades para colectar dinero que ayudara a solventar gastos hospitalarios, acompañamiento a las consultas médicas debido al fuerte temor a la discriminación (un elemento crucial para asegurar el acceso a recursos terapéuticos institucionales), apoyo en la realización de procedimientos administrativos en los periodos de emergencia, entre otros.

Como pude constatar, el cuidado que pueden asumir otras mujeres trans es limitado. Las condiciones estructurales — tales como el acceso a recursos económicos, el capital social, así como las valoraciones simbólicas en torno al VIH y el cuidado de la salud compartidas en comunidad - impactaron negativamente en la capacidad de respuesta colectiva al sufrimiento.

Algunos de los casos presentados aquí ejemplifican los procesos institucionales que existen como condiciones para la atención de la salud. Por ejemplo, en el caso de Tania y Abigail el no tener DNI y no estar inscritas en el Seguro Integral de Salud constituyeron barreras durante los periodos de emergencia.

En los cuatro itinerarios terapéuticos reconstruidos, el acceso a los servicios de salud y con ello a recursos terapéuticos institucionales se dio principalmente en el periodo de emergencia, cuando las enfermedades producto de la infección avanzada por VIH habían desmejorado significativamente su salud, tal como lo señala uno de los médicos entrevistados:

Tú las ves y ves en ellas los pacientes que tú veías en la época del 2000, cuando no había tratamiento y todo el mundo se moría. Esas pacientes tú las ves ahora con las chicas trans. Cosa que ya no hay, ya no ves eso (Manuel, médico).

Esta realidad vuelve a plantear la situación de discriminación en los servicios de salud hacia mujeres trans, la cual ha sido discutida en otros estudios (Salazar et al., 2013, 2010; Velarde, 2011; Salazar, 2009). A lo largo del trabajo de campo, recogí distintos testimonios de mujeres trans sobre experiencias 
de discriminación que ellas mismas, o conocidas suyas, habían enfrentado en los servicios de salud. En tanto los procesos de enfermedad son acontecimientos de alta vulnerabilidad física y emocional, la atención estigmatizadora o incluso la inaccesibilidad a la atención de salud se constituyen en recuerdos sumamente potentes que impactarán luego en la decisión de recurrir a los servicios públicos. Así, por ejemplo, Tania temía ir al hospital, a pesar del estado crítico en el que se encontraba, debido a que pensaba que Abigail, su amiga internada hace unos meses, había muerto a causa del maltrato de los médicos. Podemos, siguiendo a Veena Das (Ortega, 2008), preguntarnos cómo estas experiencias críticas de discriminación pueden terminar constituyéndose para las afectadas en "conocimiento envenenado"; es decir, un conocimiento de la vida que se forja en el dolor de las condiciones de exclusión y que forman parte del círculo de la violencia.

\section{La vida desordenada: una narrativa de sufrimiento en torno al VIH y la sexualidad}

La salud y su cuidado cobran sentido y ocurren en un entramado de relaciones sociales. Es desde este entramado que emergen relatos individuales, producto de una interrelación entre subjetividad y estructura social, que brindan un contexto o marco de significación sin el cual no es posible entender la experiencia particular e individual de aflicción.

A través de los testimonios y relatos individuales podemos acercarnos a narrativas culturales o discursos compartidos, desde los cuales las personas suelen dar cuenta del por qué ellos/as sufren, y el lugar que este sufrimiento tiene (o puede/debe tener) socialmente. Estas narrativas culturales estarán disponibles para que las personas elaboren sus propias experiencias, así como expresa Reddy (2001, p. 52; en Ortega, 2008, p. 25): "El sufrimiento está siempre situado antes de que sepamos de él; incluso nuestro propio sufrimiento ya está interpretado antes que lo podamos expresar".

La narratividad como un modo de aprehender y dar sentido a la realidad se vuelve "una forma discursiva con la cual moralizamos la realidad" (Mattingly, 1998; en Ortega, 2008, p. 44). A partir de estas narrativas de enfermedad y dolor es posible analizar repertorios interpretativos (Burr, 1995) que tienen las personas en relación con el sufrimiento. 
En la investigación reconstruí tres tipos de narrativas en torno al cuidado de la salud a partir de las entrevistas y conversaciones sobre el fallecimiento de los cuatro casos señalados, y en general sobre los problemas de salud que enfrentan las mujeres trans. Una primera narrativa pone el énfasis del cuidado de la salud en la responsabilidad individual; de esta manera, se atribuyen como causas de la enfermedad y el fallecimiento, por un lado, a la desidia o falta de interés personal en ocuparse de la salud, que se entiende como una actitud común y general de una "mayoría" de travestis y mujeres trans. Una segunda narrativa, vinculada a la anterior, gira en torno a la valoración positiva sobre la capacidad individual de afrontamiento, que está relacionada con la salud pero que se extiende a los problemas sociales que enfrentan cotidianamente. Un sentido de fortaleza individual emerge así de los relatos sobre formas de subsistencia y maneras de afrontar la violencia. La tercera narrativa se compone de explicaciones en torno al riesgo de infección por VIH, atribuidas a lo que llaman una "vida desordenada", común entre mujeres trans, y de la cual me ocuparé especialmente en los siguientes acápites. Llevar una "vida desordenada" es una frase que sintetiza una comprensión particular del sufrimiento, en la cual es posible analizar cómo a pesar de la exclusión social, los efectos del Estado y sus políticas de gestión del VIH, han logrado penetrar $\mathrm{y}$ anidar en las subjetividades de las mujeres trans.

\subsection{El concepto epidemiológico de "riesgo" en la gestión nacional del VIH}

Siguiendo la perspectiva de Michel Foucault en torno a la producción de saberpoder, el antropólogo Nestor Perlongher define al VIH como un dispositivo (Pelúcio y Miskolci, 2009), es decir, "un conjunto resueltamente heterogéneo que compone los discursos, las instituciones, las habilitaciones arquitectónicas, las decisiones reglamentarias, las leyes, las medidas administrativas, los enunciados científicos, las proposiciones filosóficas, morales, filantrópicas" (Agamben, 2011, p. 250). Una red de elementos inscrita en relaciones de poder que, antes de prohibir, lo que busca es la producción de verdades y la intervención en los procesos de subjetivación. Foucault desarrolló el concepto de "biopoder" como parte de su análisis sobre las estrategias de control de las poblaciones. Se trata de un poder sobre la vida, cuyos mecanismos ya no buscan el castigo, sino el control mediante la interiorización de las regulaciones por parte de los propios sujetos. Por tal motivo, la producción de subjetividades será un asunto de vital importancia en el análisis de los efectos del 
poder, y en este caso específico, de los efectos del VIH/sida como dispositivo de regulación, cuyos alcances en definitiva exceden el control de la epidemia.

El pánico moral desencadenado por el VIH a inicios de la década de 1980 reactualizó concepciones morales estigmatizadoras en torno a la homosexualidad (y en general a las prácticas sexuales y genéricas no heteronormativas), ahora bajo significados asociados a la contaminación y al exceso (Weeks, 1998). Homosexuales y otros disidentes fueron considerados vectores de la infección y transformados en una población de intervención por las políticas salubristas, constituyéndose así "identidades epidemiológicas", como efecto de una serie se procedimientos discursivos de regulación, tales como las campañas de "sexo seguro", la filiación a proyectos de intervención gestionados por ONG, y en general la promoción de la responsabilidad individual frente a la infección. En suma, subjetividades "marcadas por la autoevaluación" (Pelúcio y Miskolci, 2009, p. 131).

Si bien, el espacio es corto para desarrollar de manera extensa el abordaje tanto conceptual como institucional del VIH en el Perú, lo que busco en este breve apartado es sintetizar algunos elementos clave en la gestión pública del VIH que han emergido en las narrativas de las mujeres trans entrevistadas, tales como el concepto de "riesgo" y de "estilos de vida" (Yon, 2014; Nichiata et al., 2008; Menéndez, 1998). A través de estos conceptos, se han articulado los mensajes y los esfuerzos preventivos del VIH a nivel nacional, los cuales no han variado significativamente en el transcurso de las últimas dos décadas de respuesta nacional a la epidemia (Cáceres y Mendoza, 2009). Estas prácticas institucionales de gestión del VIH han concentrado sus esfuerzos principalmente en las llamadas "poblaciones clave", entre las cuales figuran los "hombres que tienen sexo con otros hombres" (HSH) y hasta no hace mucho las mujeres trans (antes consideradas también bajo el acrónimo de HSH).

Si bien la epidemiología desde la salud pública, como saber experto, tiene distintas vertientes, de acuerdo con el antropólogo argentino Eduardo Menéndez (1998, p. 39) es más bien una vertiente "positivista" la hegemónica en "los aparatos sanitarios de América Latina y en la mayoría de sus institutos". Bajo esta perspectiva, son los factores biológicos los preponderantes en la reproducción de las enfermedades e infecciones, y en segundo lugar (o secundariamente) se encontrarían los aspectos sociales y culturales.

El riesgo se define como "la probabilidad de los individuos de enfermar o morir asociadas a ciertos comportamientos, situaciones o características" (Yon, 2014, p. 119). El no uso de preservativo en una relación sexual es consi- 
derado un comportamiento individual de riesgo sexual. Otros comportamientos individuales que se consideran de riesgo desde los estudios epidemiológicos, y que en combinación incrementan la posibilidad de transmisión del VIH, son el uso alcohol en las relaciones sexuales (dado que puede inhibir el uso de condón) y el número de parejas sexuales (dado que abre una posibilidad mayor de transmisión), entre otros. Desde este concepto epidemiológico, ciertas colectividades son consideradas "grupos de riesgo", y los individuos asociados a las situaciones definidas bajo "el riesgo" terminan siendo asimilados a este grupo (Nichiata et al., 2008).

Vinculado al riesgo, el concepto de "estilos de vida" ha sido usado en el abordaje de la infección. De acuerdo con Menéndez (1998), el concepto parte de la premisa que, a través del comportamiento de los individuos, es posible observar la cultura de un grupo social. De esta manera, ciertos comportamientos fueron aislados de su contexto cultural e ideológico más amplio y considerados como "riesgosos", siendo además adjudicados a la responsabilidad individual.

Las estrategias de prevención del VIH en el país, a diferencia de otras latitudes, se han restringido al abordaje individual en la dinámica de infección (en torno a las actitudes, conocimientos y prácticas de los grupos con mayor número de casos). Esta comprensión particular de la infección de VIH guarda relación con un abordaje neoliberal de la salud, en el que esta se ha ido considerando como un campo individual y privado, y en donde las posibilidades de alivio recaen en lo que se considera principalmente la voluntad y los recursos de quien padece la enfermedad (Menéndez, 2009).

\subsection{Vida desordenada: irresponsabilidad como causa atribuidas a la infección por $\mathrm{VIH}$}

[...] hacen su vida un desorden, toman... Será por la misma cultura, ¿no?, que ignorantemente... Pues ellas dirán: “Ay, no, al final todas vamos a morir”. Entonces pienso así que a ellas no les importa: se van, bailan, toman, lo poco que ganan pues a la semana [...]. Ya están en la discoteca tomando con los chicos. Entonces esa vida hacen ellas: una vida libre, o sea, como diciendo que no les importa, y peor aún sin la familia. Si no son allegados a su familia, son rechazadas. Peor, pues (Olga, mujer trans).

Cuando en el transcurso de las entrevistas preguntaba por qué consideraban que las mujeres trans se enfermaban y fallecían, varias me compartían relatos parecidos a la cita anterior. "Una vida desordenada" o simplemente "desor- 
den" es la síntesis de una trayectoria marcada por la irresponsabilidad individual que finaliza en muerte. Por ejemplo, en la explicación de Olga, una mujer trans perteneciente a la red de cuidado de una de las fallecidas, llevar una vida desordenada implica una serie de acciones como beber licor en grandes cantidades, salir y desvelarse, gastar el dinero ganado en diversión en vez de invertirlo en alguna otra cosa.

En este tipo de narrativa, la posibilidad de cambiar el curso de los hechos reside únicamente en la mujer trans. Así, por ejemplo, en la siguiente cita, la desidia es la explicación ofrecida para entender por qué las mujeres trans fallecen de infecciones asociadas al VIH:

En el caso de las chicas que fallecen, yo tengo un simple concepto de eso: que la persona que fallece puede ser de esa enfermedad [VIH/sida] o de otra índole, la cosa es que fallece por su desidia, no necesariamente la enfermedad la está matando. Es desidia porque ellas saben que tienen que curarse [...]. Encima que no se cuidan empiezan a desbandarse, tomar, drogas, relaciones sexuales sin protección. Entonces eso complica más las cosas (Viviana, mujer trans).

De manera general, y tal como se puede observar en ambas citas, el posicionamiento de la narradora que emerge en el relato la ubica en una posición externa a lo que cuenta (ella "no" tiene una vida desordenada), con la autoridad de quien puede ver claramente el panorama y emitiendo así un juicio moral sobre lo que "otras" hacen. Tanto en la explicación del "desorden" como del "orden" las mujeres trans aparecen de manera homogénea como un sujeto colectivo representado a partir de sus prácticas "de desorden".

Esto hemos visto anteriormente. Tal narrativa puede entenderse como una apropiación que se hace de las nociones epidemiológicas de riesgo y estilos de vida, a través de las cuales se ha buscado controlar la epidemia del VIH en poblaciones con alta prevalencia de infección, como son las mujeres trans. Mediante el uso de un repertorio interpretativo que alude a estas explicaciones epidemiológicas se construye una narrativa moral en torno a la responsabilidad de infección y curación.

Asimismo, en esta narrativa se pone de manifiesto la agencia discursiva de las mujeres trans a través de una diferenciación activa de una idea de colectivo, definido negativamente y homogéneamente por un sentido de irresponsabilidad frente a la salud. Así, el carácter moral de las narrativas supone el posicionamiento de las personas que elaboran estos relatos ante ellas y ante mí como interlocutor. 


\section{Reflexiones finales}

La recurrencia de la infección de VIH y muerte por enfermedades asociadas - la cual es perversamente común a las mujeres trans- puede ser interpretada desde el marco conceptual propuesto, como el resultado de procesos de producción de sufrimiento y vulnerabilidad social. Al hablar de "producción" hacemos énfasis en la dinámica activa de distintos factores que han limitado el acceso y ejercicio de la salud. A partir del análisis de las trayectorias de enfermedad y contextos de fallecimiento, se ha intentado desarrollar cómo la vulnerabilidad social a la enfermedad en el caso de las mujeres trans es activamente producida a partir de privaciones, restricciones e indiferencia institucional y social.

Uno de los aportes al debate que este estudio quiere ofrecer es la comprensión de los efectos de la exclusión y el manejo institucional del VIH/sida en las subjetividades de las mujeres trans, los cuales han sido abordados a través del análisis de la comunidad moral y las narrativas de sufrimiento. Busco así discutir la reproducción del sufrimiento social vinculado a la vivencia del VIH en contextos de exclusión desde las valoraciones morales y las narrativas transmitidas en comunidad.

En la narrativa de la "vida desordenada", un orden moral se revela a través de valoraciones sobre la noción de responsabilidad individual de personas con identidades de género y comportamientos sexuales estigmatizados. El excesivo peso que se da a la capacidad individual no es casual. Siguiendo a Veena Das, el énfasis en la responsabilidad individual en el abordaje de la salud debería pensarse como parte de una teodicea secular a través de la cual las fuerzas sociales y políticas que infringen sufrimiento (por ejemplo, la exclusión social activamente producida y reproducida) aparezcan mitigadas.

En la vida desordenada no solo "se culpa a la víctima" bajo el precepto moral neoliberal de la responsabilidad individual, sino que actualiza y mantiene activo un discurso que alude al control salubrista que homogeniza la experiencia de vivir con VIH de las mujeres trans en términos de exceso, descontrol y riesgo. La narrativa pone de manifiesto los efectos del control del VIH, que como dispositivo de biopoder en el control de la sexualidad y la salud ha penetrado con efectividad en la subjetividad de las mujeres trans, produciendo un sentido de sí en relación con un "nosotras" colectivo definido por el exceso y el riesgo. Como está claro, su efectividad no radica en lograr una disminución de la prevalencia del VIH entre las mujeres trans, como las 
políticas de Estado pudiesen pretender, sino en su capacidad de haberse constituido como un campo semántico y discursivo potente de subjetivación.

El discurso actual del cuidado individual de la salud se vuelve así un campo activo de negociación y de diferenciación social, que es usado por varias de las entrevistadas para tomar distancia de aquel colectivo definido en términos negativos por el riesgo. De esta manera, un efecto del discurso del riesgo individual en el campo de la gestión y control del VIH, y en el contexto más amplio de exclusión social de las personas trans, ha sido fragilizar aún más el sentido de comunidad a través de los procesos de estigmatización y de culpabilización que se dan entre mujeres trans y que hemos visto de manera particular en algunos contextos de fallecimiento aquí descritos.

Finalmente, me gustaría proponer que el posicionamiento discursivo presente en las entrevistadas, y que hasta aquí he abordado como un recurso de diferenciación en el marco de un discurso de responsabilidad individual frente al VIH, es probablemente solo una de las maneras de interpretar las palabras (y sus sentidos) de mis interlocutoras. Leyendo los agudos comentarios de la activista travesti argentina Lohana Berkins (2004) sobre cómo la exclusión social y el hacinamiento no permiten que las mujeres trans se reconozcan a sí mismas como sujetas particulares, con una historia particular, con gustos y sueños propios, encontré que las travestis y mujeres trans podrían estar sugiriendo una actitud frente a la exclusión que no se agota en la diferenciación frente al colectivo de "mujeres trans" definido por homogenización, sino que podría ser también un reclamo de individualidad, de singularidad. Desde esa perspectiva, el posicionamiento individual de desmarcarse de un colectivo definido por el "desorden" y moralmente negativo resultaría en un ejercicio particular de la agencia mediante el cual se haría frente a los efectos de homogenización que produce la exclusión social, y en particular un discurso y gestión del VIH que continúa articulándose en torno al concepto y la retórica del riesgo individual, sin realmente hacer frente a las privaciones y violencias institucionales que producen vulnerabilidad y fragilizan la capacidad de respuesta ante la adversidad de travestis y mujeres trans. 


\section{Bibliografía}

Agamben, G. (2011) ¿Qué es un dispositivo? Sociológica, 26(73), 249-264.

Anderson, J. (2002). Narrativas de aflicción de mujeres en la sierra del Perú. Alpanchis, XXXI(56), 75-106.

Berkins, L. (2004). Eternamente atrapadas por el sexo. En Fernández, J., D’uva, M. y Viturro, P. Cuerpos ineludibles (pp. 19-24). Buenos Aires: Ediciones de Ají de Pollo.

Berkins, L. (Ed.) (2007). Cumbia, copeteo y lágrimas. Buenos Aires: A.L.I.T.T.

Brown, P., Barret, R. y Padilla, M. (1998). What Is Medical Anthropology? En Brown, P. J. (Ed.) Understanding and Applying Medical Anthropology (pp. 10-19). Nueva York: Praeger.

Burr, V. (1995). What Does It Mean to be a Person? An Introduction to Social Constructionism. Londres: Routledge.

Butler, J. (2010). Marcos de guerra: las vidas lloradas. Buenos Aires: Paidós.

Cáceres, C. y Mendoza, W. (2009). The National Response to the HIV/AIDS Epidemic in Peru: Accomplishments and Gaps, a Review. J Acquir Immune DeficSyndr, 51, supl. 1, S60-6.

Comisión Interamericana de Derechos Humanos (2015). “En el Día Internacional de la Memoria Trans, CIDH urge a los Estados a aumentar la expectativa de vida de las personas trans en América". Comunicado de prensa. Tomado de http://www.oas.org/ es/cidh/prensa/comunicados/2015/137.asp. Revisado 05-08-17.

Cosme, C., Jaime, M., Merino, A. y Rosales, J. (2007). La imagen indecente. Diversidad sexual, prejuicio y discriminación en la prensa escrita peruana. Lima: Instituto de Estudios Peruanos.

Defensoría del Pueblo (2016). Derechos humanos de las personas LGBTI: necesidad de una política pública para la igualdad en el Perú. Informe Defensorial 175.

Farmer, P. (2007). Una antropología de la violencia estructural. El caso de Haití. Temas, 52, 63-73.

Figueiredo, A. (2011). "Se pudesse ressurgir, viria como o vento". Narrativas da dor: corporalidade e emocoesna experiencia da travestilidade. Sexualidad, Salud y Sociedad. Revista Latinoamericana, 8, 90-112.

Foucault, M. (2005). Historia de la sexualidad. Tomo I: La voluntad de saber. Madrid: Siglo XXI Editores.

Goffman, E. (1995). Estigma, la identidad deteriorada. Buenos Aires: Amorrortu Editores. 
Good, B. (2003). El cuerpo, la experiencia de la enfermedad y el mundo vital: una exposición fenomenológica del dolor crónico. Medicina, racionalidad y experiencia. Una perspectiva antropológica (pp. 215-246). Barcelona: Ediciones Bellaterra.

Jaime, M. (2011). Informe anual sobre Derechos humanos de personas trans, lesbianas, gays y bisexuales en el Perú 2010. Lima: Centro de Promoción y Defensa de los Derechos Sexuales y Reproductivos PROMSEX-Red Peruana TLGB.

Kleinman, A., Das, V., y Lock, M. (1997). Social Suffering. California: University of California.

Menéndez, E. (2009). De sujetos, saberes y estructuras. Introducción al enfoque relacional en el estudio de la salud colectiva. Buenos Aires: Lugar Editorial.

Menéndez, E. (1998). Estilos de vida, riesgos y construcción social. Conceptos similares y significados diferentes. Estudios Sociológicos, XVI, 46.

Ministerio de Salud. (1999). Mujeres de negro: la muerte materna en zonas rurales del Perú. Estudio de casos. Lima: MINSA, Proyecto 2000.

Ministerio de Salud (2014). Análisis de la situación epidemiológica del VIH-SIDA en el Perú, 2013. Lima: MINSA.

Montalvo, J., Andía, B. y Rodríguez, R. (2009). Realidades invisibles. Violencia contra travestis, transexuales y transgéneros que ejercen comercio sexual en la ciudad de Lima. Lima: Runa Instituto de Desarrollo y Estudios sobre Género.

Nichiata, L., Bertolozzi, M. R., Takashi, R. y Fracolli, L. A. (2008). A utilizacao do conceito "vulnerabilidade" pela enfermagem. Rev. Latino-am Enfermagem, 16(5).

Núnez-Curto, A. (2018). Muerte, enfermedad y vulnerabilidad social: narrativas y prácticas de cuidado en los contextos de muerte de las mujeres trans en Lima (tesis de maestría en Antropología). Lima: Pontificia Universidad Católica del Perú.

Ortega, F. (2008). Veena Das: sujetos del dolor, agentes de dignidad. Bogotá: Universidad Nacional de Colombia, Facultad de Ciencias Humanas, Centro de Estudios Sociales (CES).

Nussbaum, M. (2006). El ocultamiento de lo humano. Repugnancia, vergüenza y ley. Buenos Aires: Katz.

Pelúcio, L. y Miskolci, R. (2009) A prevenção do desvio: o dispositivo da aids e a repatologização das sexualidades disidentes. Sexualidad, salud y sociedad. Revista Latinoamericana, 1, 125-157.

Ramírez, B. (Coord.) (2012). Informe anual sobre derechos humanos de personas trans, lesbianas, gays y bisexuales en el Perú 2011. Lima: Centro de Promoción y Defensa de los Derechos Sexuales y Reproductivos-PROMSEX, Red Peruana LGBT. 
Salazar, X. y Villayzán, J. (2009). Lineamientos para el trabajo multisectorial en población trans, derechos humanos, trabajo sexual y VIH/Sida. Lima: IESSDEH, UNFPA, Red LacTrans.

Salazar, X., Maguiña, J. L., Villayzán, J., Anamaría, P., Sandoval, C., ..., Cáceres, C. (2013). “...Y me di cuenta de que el sida no es sinónimo de muerte”. Diagnóstico del acceso a servicios y programas de prevención de salud sexual y reproductiva de las personas viviendo con VIH. Lima: UPCH, IESSDEH, Peruanos Positivos, Red Nacional por los derechos de las personas Travesti, Transgénero, y Transexuales del Perú.

Salazar, X., Villayzán, J., Silva-Santisteban, A. y Cáceres, C. (2010). las personas trans y la epidemia del VIH/sida en el Perú: aspectos sociales y epidemiológicos. Lima: IESSDEH, UPCH, ONUSIDA, amFAR.

Scheper-Hughes, N. (1997). La muerte sin llanto. Violencia y vida cotidiana en Brasil. Madrid: Ariel.

Scheper-Huges, N. y Lock, M. (1987). The Mindful Body: A Prolegomenon to Future Work. Medical Anthropology Quaterly, 1(1), 6-41.

Sosa, C. (2019). Las malas. Buenos Aires: Editorial Tusquets.

Velarde, Ch. (2011). La igualdad en la lista de espera. Necesidades, barreras y demandas en salud sexual, reproductiva y mental en población trans, lesbiana y gay. Lima: PROMSEX.

Weeks, J. (1998) Sexualidad. Ciudad de México: Paidós.

Yon, C. (2014). Vulnerabilidad social, salud y derechos sexuales de adolescentes ayacuchanos. En Barrantes, R. y Buse, P. (Eds.). Salud, vulnerabilidades, desigualdades (pp. 115-140). Lima: Instituto de Estudios Peruanos. 